\title{
Freestanding 3D Supramolecular Particle Bridges: Fabrication and Mechanical Behavior
}

\author{
Xing Yi Ling, In Yee Phang, Holger Schönherr, David N. Reinhoudt, \\ G. Julius Vancso, and Jurriaan Huskens*
}

\begin{abstract}
$\boldsymbol{F}_{\text {reestanding particle bridges with controlled composition and macroscopic }}$ robustness are demonstrated by the use of supramolecular nanoparticle assembly. Self-assembly of nanoparticles, templating, and supramolecular glue infiltration are combined to form stable and ordered three-dimensional polystyrene particle composites on a polydimethylsiloxane stamp. Freestanding hybrid polystyrene nanoparticle bridges are obtained by transfer printing of the hybrid structures onto topographically patterned substrates via host-guest interactions. The mechanical robustness and rigidity of the particle bridges can be controlled by manipulating the layer-by-layer cycles of supramolecular glues of gold nanoparticles and dendrimers. Atomic force microscopy-based microbending results, in particular the location and force-dependent deflection behavior, confirm that the particle bridge fulfills the classical supported-beam characteristics. As estimated from classical beam theory, the bending moduli of the particle bridges vary between 0.8 and $1.1 \mathrm{GPa}$, depending on the degree of filling by the supramolecular glues. Failure analysis on the particle structure indicates linear elastic behavior and a plastic deformation upon failure.
\end{abstract}

\author{
Keywords: \\ - atomic force microscopy \\ - dendrimers \\ - host-guest systems \\ - nanoparticles \\ - self-assembly
}

\section{Introduction}

The self-assembly of nanoscale building blocks into wellcontrolled structures with active material properties and their

[*] Prof. J. Huskens, Dr. X. Y. Ling, ${ }^{\dagger}$ Prof. D. N. Reinhoudt Molecular Nanofabrication Group MESA + Institute for Nanotechnology University of Twente $7500 \mathrm{AE}$, Enschede (The Netherlands) E-mail: J.Huskens@utwente.nl

Dr. I. Y. Phang, ${ }^{\dagger}$ Prof. H. Schönherr, ${ }^{+}$Prof. G. J. Vancso Materials Science and Technology of Polymers Group MESA+ Institute for Nanotechnology University of Twente, $7500 \mathrm{AE}$, Enschede (The Netherlands)

[+] Present address: Department of Physical Chemistry University of Siegen

Adolf-Reichwein-Str. 2, AR-F0103, 57076 Siegen (Germany)

[†] These authors contributed equally to this work.

․ Supporting Information is available on the WWW under http:// www.small-journal.com or from the author.

DOI: $10.1002 /$ smll.200900068 precise positioning are two major technical challenges in the ongoing miniaturization of electronic devices. ${ }^{[1]}$ The design and fabrication of electronic devices generally involve freestanding nanostructures with well-controlled architectures partially coupled to the supporting substrate. The freely suspended nanostructures can also act as connections between two supporting structures. The conventional preparation of these nanostructures generally involves sophisticated cleanroom lithography techniques. ${ }^{[2-4]}$ Alternative preparation methods of freestanding nanostructures for other applications, with controlled composition and macroscopic robustness, have been explored. Most reports focus on the synthesis of freestanding nanocomposite cantilevers ${ }^{[5]}$ and membranes, ${ }^{[6]}$ polymer layer-by-layer (LbL) thin films, ${ }^{[7]}$ and polymer films with added metallic ${ }^{[8-10]}$ or inorganic fillers, ${ }^{[11-14]}$ which enhance the mechanical stability.

Our group utilizes supramolecular chemistry, that is, the complexation of $\beta$-cyclodextrin (CD) and its guest molecules such as adamantane and ferrocene, to assist the formation of particle structures. ${ }^{[15,16]}$ Patterned nanoparticle structures on surfaces were obtained by direct adsorption of supramolecular guest- and host-functionalized nanoparticles ${ }^{[17]}$ onto (patterned) CD self-assembled monolayers (SAMs) through 
multivalent host-guest interactions and LbL assembly. ${ }^{[16,18-21]}$ Recently, we have demonstrated the fabrication of stable, three-dimensional (3D), freestanding hybrid particle crystals by a sequential process that comprises bottom-up selfassembly, supramolecular host-guest chemistry, and transfer printing. ${ }^{[22]} \beta$-CD-functionalized polystyrene (PS-CD) nanoparticles were organized and shaped into desired architectures by using patterned polymer templates with predefined geometries and dimensions. Supramolecular host-functionalized gold nanoparticles and guest-functionalized dendrimers $^{[23]}$ function as supramolecular glues to chemically connect individual PS particles into integrated hybrid particle structures by LbL assembly. A new type of freestanding hybrid particle bridge was obtained by transfer printing of the structures, through conformal contact, onto a topographically patterned CD-functionalized target substrate. The PS-CD particles in these bridges are connected by multiple molecules and metallic nanoparticles through intrinsically weak hostguest interactions to form robust and macroscopically stable microbridges.

The determination of the mechanical properties of a submicrometer material is important in understanding the physical and structural properties of this material for its potential application in micro/nanoelectromechanical systems (M/NEMS). ${ }^{[24,25]}$ Atomic force microscopy (AFM), as one of the most commonly used scanning probe microscopy techniques, has become an eligible instrument for micro/nanoscopic mechanical measurements. Owing to its high lateral resolution (typically $\approx 5-20 \mathrm{~nm}$ ) and its wide range for high-precision force measurements $(\approx 10 \mathrm{pN}$ to $\approx 10 \mu \mathrm{N})$, AFM has been widely exploited to measure the mechanical properties of a wide range of nanomaterials. ${ }^{[26]}$

The microbending test is one of the AFM-based measurement techniques that allow the determination of mechanical responses of nanomaterials. A variety of long and freely suspended nanostructures, such as carbon nanotubes, ${ }^{[26]}$ nanowires, ${ }^{[27]}$ and peptide nanotubes, ${ }^{[28]}$ drop-cast on micropatterned substrates or porous membranes have been subjected to bending tests. ${ }^{[28-31]}$ The deformation of the suspended nanostructures was monitored by applying different forces during AFM imaging or by AFM-based force spectroscopy at the center of the suspended nanostructures. ${ }^{\text {[28-32] }}$ The loading force exerted on the suspended nanostructures can be controlled over a wide range by a selection of probe sensors with different stiffnesses. Thus, the bending modulus of a given (nano)material can be estimated from the experimentally determined force-deformation relationships. ${ }^{[28-30,33]}$

Herein, we report the fabrication and investigation of the nanomechanical properties of the unique freestanding hybrid particle bridges introduced above by AFMbased microbending tests. AFM is used not only to directly determine the mechanical properties by bending tests, but also to interrogate the structural properties and the integrity of the 3D particle bridges. In particular, the dependence of the micro- mechanical properties on the number of layers in the $\mathrm{LbL}$ assembly step and on the length of the hybrid particle bridges has been addressed.

\section{Results and Discussion}

The ability to precisely assemble individual particles into desired geometries and to bind them into stable 3D architectures is important for their practical application. A sequential process for the fabrication of stable, freestanding 3D supramolecular particle structures on a topographically patterned support was developed by combining the bottom-up self-assembly of particles, supramolecular LbL assembly, and transfer printing. ${ }^{[22]} \mathrm{CD}$-functionalized particle crystals were first convectively assembled on a patterned elastomeric polydimethylsiloxane (PDMS) stamp (Scheme 1). Supramolecular LbL assembly of specific guest- (adamantyl (Ad) dendrimer) and host-functionalized (Au-CD) supramolecular glues within the particle crystal effectively integrates the crystal into a single building block. The preassembled linear 3D particle crystals were brought into conformal contact with a topographically patterned CD SAM surface. By transfer printing of the complete structures, 3D freestanding particle bridges were obtained. ${ }^{[22]}$

Figure 1A-D shows an as-prepared convectively assembled particle crystal infiltrated with Ad dendrimers only, and hybrid particle crystals after 10, 20, and $30 \mathrm{LbL}$ assembly cycles. A particle crystal with a relatively smooth surface was first obtained from convective assembly and dendrimer infiltration (Figure 1A). With increase in the number of bilayers, more material was deposited within the crystal as well as on the nanoparticle surface. Particularly in the case of 20 and $30 \mathrm{LbL}$ assembly cycles, the supramolecular material was observed bridging adjacent nanoparticles (Figure 1C and D). The supramolecular LbL assembly of Ad dendrimers and Au-CD within the PS-CD crystal resulted in the crosslinking of individual nanoparticles into a 3D stable nanoparticle crystal, with full retention of the order achieved during the convective assembly. The voids between the particles became smaller owing to filling by the supramolecular glues. In addition, the surface of the nanoparticles became rougher (Figure 1E), with occasional agglomerates (typical size, tens of nanometers) attached to the surface of the
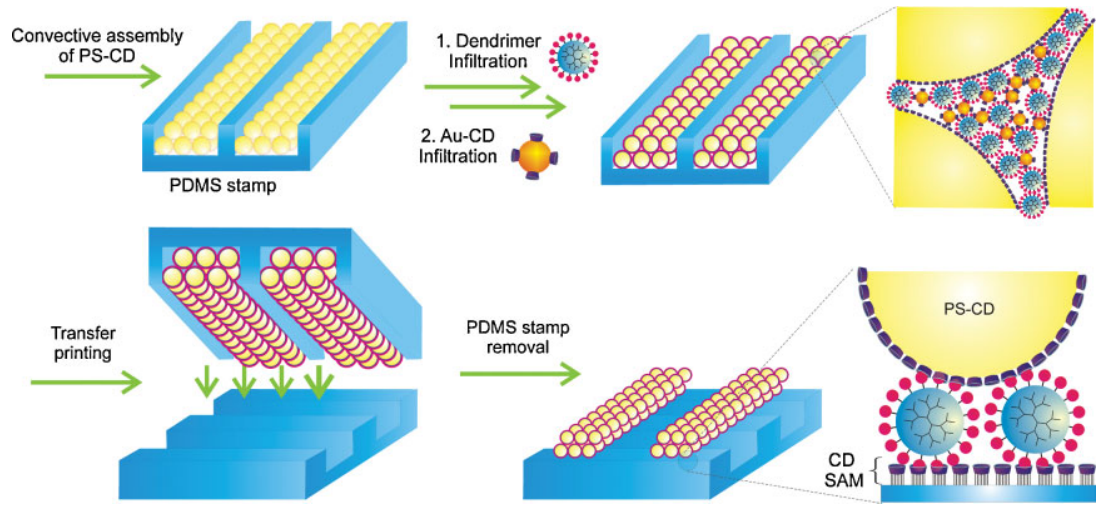

Scheme 1. Preparation of the hybrid particle bridges on a topographically patterned CD SAM. 

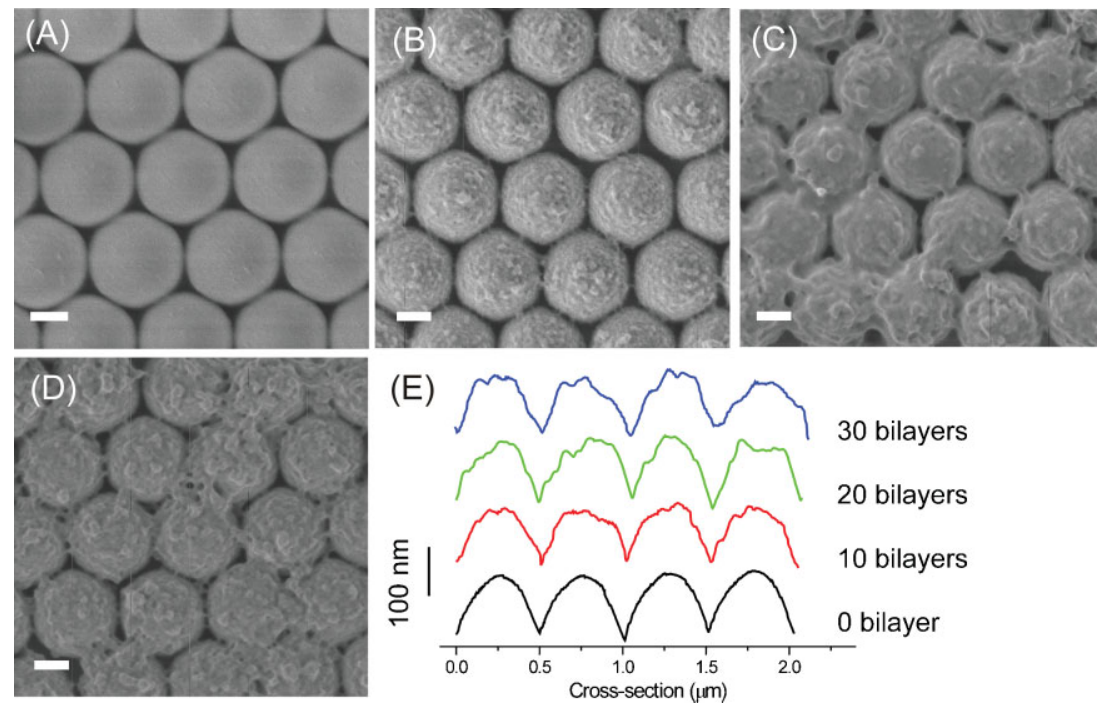

Figure 1. A-D) SEM images of a particle crystal infiltrated with Ad dendrimers only (A), and after 10 (B), 20 (C), and 30 (D) LbL assembly cycles of Ad dendrimers and Au-CD within the nanoparticle crystals. Scale bars: $200 \mathrm{~nm}$. E) AFM cross-sectional profiles of the particle crystals with increasing numbers of bilayers.
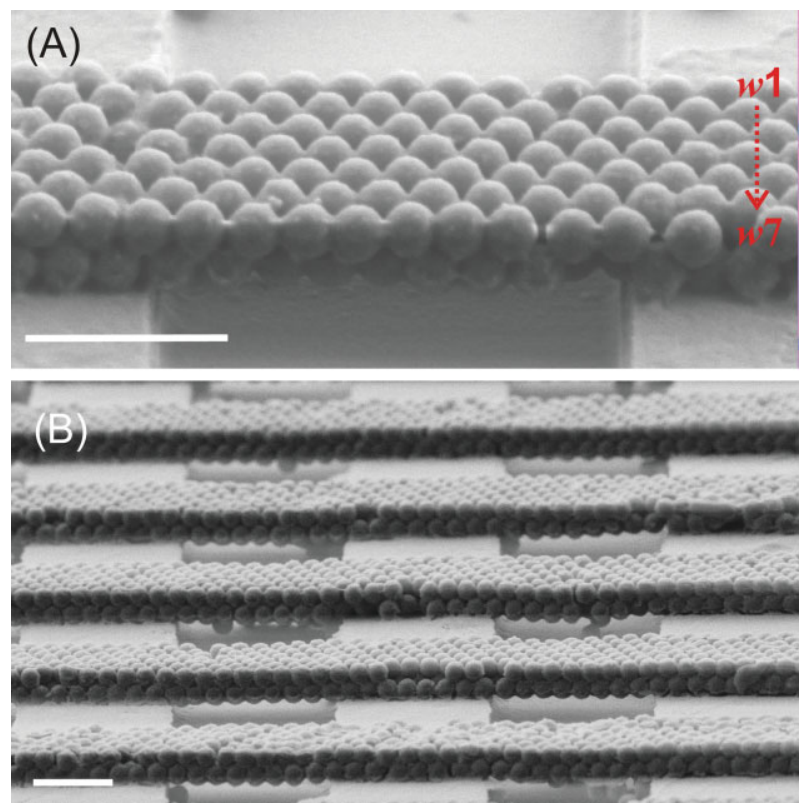

Figure 2. A,B) SEM images of the freestanding hybrid particle structures ( 30 bilayers of Ad dendrimer and Au-CD nanoparticles deposited by $\mathrm{LbL}$ assembly) on a topographically patterned $\mathrm{CD} \mathrm{SAM}$ on a $\mathrm{SiO}_{2}$ substrate. Scale bars: $2 \mu \mathrm{m}$; w1-w7: position of the particles along the width.

PS-CD particles. The presence of these agglomerates indicates the intermixing of layers of supramolecular glues during the assembly process.

Figure 2 shows scanning electron microscopy (SEM) images of 3D freestanding hybrid particle bridges (width $=3 \mu \mathrm{m}$ ), transfer printed on a topographically patterned CD SAM surface with $4-\mu \mathrm{m}$-wide grooves. The particle bridges rest on the supports in an orthogonal fashion, forming supported microbridges. Remarkably, the structures were found to be suspended in a beamlike configuration above the support at two ends in the absence of stress, thus indicating considerable mechanical stiffness within the particle bridge.

The conventional LbL assembly of oppositely charged polyelectrolytes generally produces nanometer-thick coatings via attractive electrostatic forces between the polyelectrolytes. ${ }^{[34]}$ In the supramolecular LbL assembly used here, guest-functionalized organic molecules and host-functionalized metallic nanoparticles provide thin, stable coatings on the PS-CD particle crystal surface. Most importantly, the constituents act as complementary supramolecular glues that strengthen the cohesion between the individual PS-CD particles through thermodynamically stable multivalent host-guest interactions. ${ }^{[19,35]}$

The effect of the degree of filling by supramolecular glues on the stability of the particle crystal was examined by using hybrid particle crystals assembled by 10, 20, and 30 LbL cycles of supramolecular glues. All assemblies yielded microbridges that spanned across the micrometer-wide grooves of the supports (Figure S1, Supporting Information). However, more defects and larger numbers of loosely bound particles were found on particle bridges prepared with fewer LbL cycles. The results indicate that the mechanical robustness and rigidity of the particle microbridges can be modulated by the use of nanometer-sized organic and metallic supramolecular glues with different layer thicknesses.

Subsequently, AFM-based microbending tests were performed on the obtained microbridges. A stiff cantilever $\left(k_{\mathrm{c}} \approx 28 \mathrm{~N} \mathrm{~m}^{-1}\right)$ was used in this study because of the high loading force required to deflect the particle bridges. As indicated in Scheme 2A, the particle bridge is deflected by a point load subjected by the AFM tip. A "point-and-shoot" function, controlled by an $X Y Z$ closed-loop feedback system, was adopted to predetermine the point of contact at the center of each individual particle during the force measurement. The deformation of the particle bridge, generally in the range of tens of nanometers, was recorded to give a corresponding force-displacement curve. In addition, tapping-mode AFM images of the bridges were captured in situ before and after each bending test. Therefore, direct comparison of the morphology was made to assess the effect of each loading force.

The overall mechanical response of a particle bridge across the spanned length $(L)$ upon a point load $(F)$ of $5 \mu \mathrm{N}$ (Scheme $2 \mathrm{~A}$ ) is demonstrated in a force-deflection curve (Figure $3 \mathrm{~A}$; see Supporting Information for details on the AFM data conversion). For clarification, the PS particles across the bridge were numbered as indicated in Scheme 2A. The forcedeflection curves show an increase in the deflection for particles located closer to the center of the bridge at a force of $5 \mu \mathrm{N}$. In all force-deflection curves, the smallest slope was always observed at the middle of the freely standing particle 
(A)

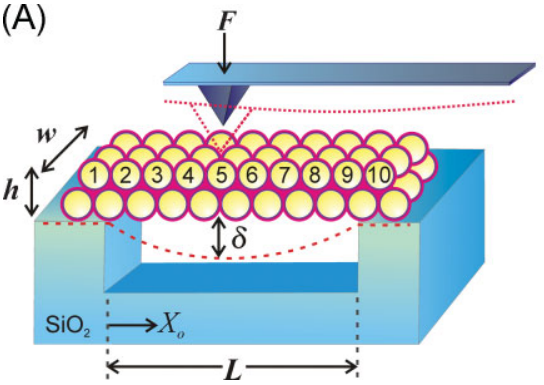

(B)

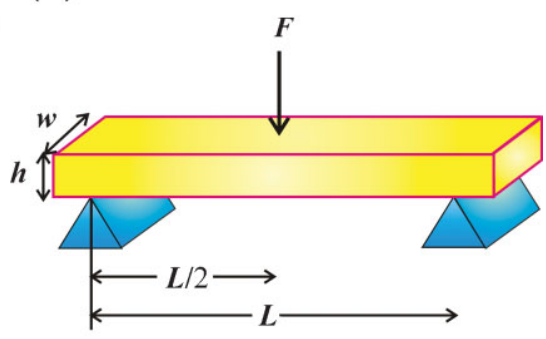

Scheme 2. A) Schematic of the bending test performed on a particle bridge by AFM. $w$ and $h$ denote the width and thickness of the supported particle bridge, $L$ is the spanned length of the freestanding structure, $F$ represents the applied loading force, and $\delta$ is the deflection of the bridge under load. B) A continuous beam supported at both ends.
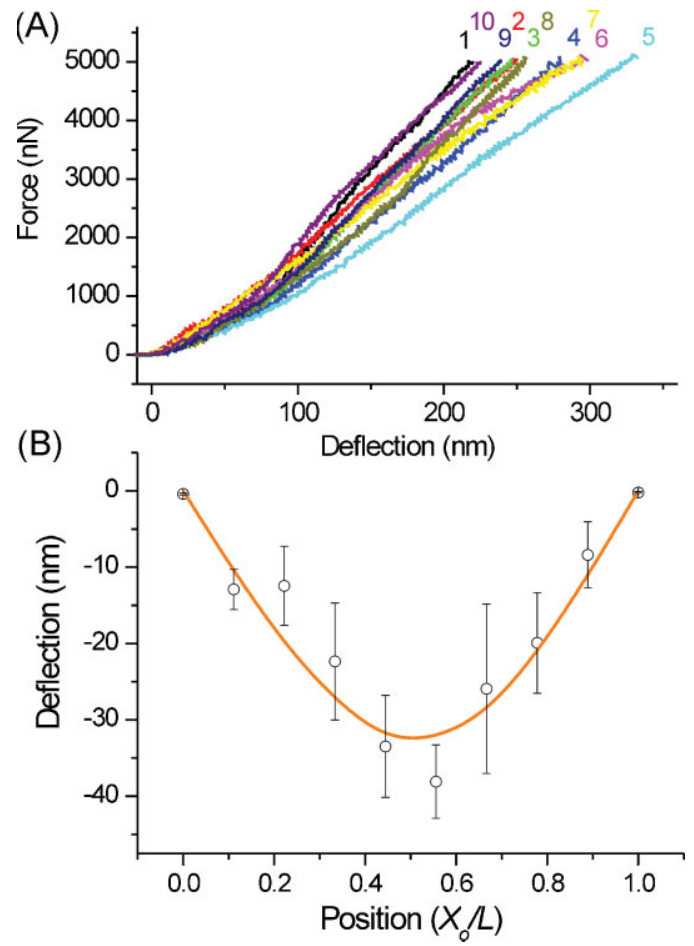

Figure 3. A) Force-deflection curve of a particle composite bridge (infiltrated with $30 \mathrm{LbL}$ cycles, $L=4 \mu \mathrm{m}$ ) across its spanned length. The PS particles across the bridge are numbered 1-10. B) General deflection-location curve obtained from eight different particle bridges (infiltrated with $30 \mathrm{LbL}$ cycles) at $5 \mu \mathrm{N}$. The deflection-location curve was fitted with classical beam theory with a point load at the center.

structure, while the highest slope was recorded for particles residing on the silicon substrate.

To measure the bending modulus of the particle bridge, the deflection of the bridge was corrected for the indentation of the AFM tip into a PS particle residing on the silicon substrate (Figure $3 \mathrm{~A}$, curve 1). In addition, to determine the general deflection behavior of the particle bridges and the accuracy and reproducibility of the bending test, the deflection curves obtained on eight different particle bridges are summarized in Figure 3B. The data were plotted against $X_{\mathrm{o}} / L$, where $X_{\mathrm{o}}$ is the location of the applied force along the beam and $L$ the total spanned length. The summarized deflection curve displays a highly reproducible deflection behavior of the freestanding

structures attached by two pivots at its ends. The deflection curve is symmetrical and exhibits a maximum deflection at the center of the particle bridge. All these characteristics are in accordance with a typical supported beam (Scheme 2B).

Figure 4 shows AFM height images and cross-sectional height profiles of a hybrid particle bridge acquired before and after a bending test at $F=5 \mu \mathrm{N}$. Prior to the bending test, the particle bridge is level and horizontally lying on two supports (Figure $4 \mathrm{~A}$ and $\mathrm{C}$ ). After subjecting the bridge to $5 \mu \mathrm{N}$ point loads across the particle bridge, indentation marks on the PS particles were clearly observed (Figure 4B). Despite the indentation marks and deflection during bending, the particle bridge returned to its original unstressed state after the bending test (Figure 4C), that is, no plastic deformation of the particle bridge was observed. The hybrid particle composite bridges, fabricated bottom-up from self-assembled individual particles and supramolecular glue, thus displayed a robust mechanical strength that is commonly observed in bulk and homogeneous materials.

The classical beam theory is the most frequently used model to quantitatively describe the deformation of a material upon bending. ${ }^{[30]}$ It is derived from Hooke's law, and relates the midpoint applied load $(F)$ and the maximum deflection $(\delta)$ of a rectangular beam as Equations (1) and (2):

$F=k \delta$

$\Rightarrow F=\frac{48 \times I \times E}{L^{3}} \delta$

where $k$ is the material spring constant, $E$ is the bending modulus of the material, $I=\left(w h^{3}\right) / 12$ is the moment of inertia, $w$ and $h$ denote the width and thickness of the beam, respectively, and $L$ is the spanned length of the freestanding structure (Scheme 2). Several criteria must be fulfilled for the application of the classical beam theory: 1) the supported beam is initially straight and unstressed;2) the material of the beam is linearly elastic and homogeneous; and 3) the cross section remains the same before and after the bending test.

The applicability of the classical beam theory in the determination of the bending modulus of the particle bridges is supported by the SEM and AFM images in Figures 2-4. The SEM image confirms that the bridges appeared straight before bending. The AFM data obtained prior to and after bending indicate that the structures exhibited elastic deformation and remained unchanged (Figure 4A and B). The deflection curve (Figure 3B) exhibited a classical position-dependent behavior of a single-beam bridge, with the maximum deflection observed at the midpoint of the bridge. All these results confirm that the deformation behavior of the particle bridge can be described by Equation (2).

The 3- $\mu \mathrm{m}$-wide particle microbridge in Figure $2 \mathrm{~B}$ is composed of seven rows of discrete 500-nm particles. Therefore, we determined the mechanical properties of the bridges 

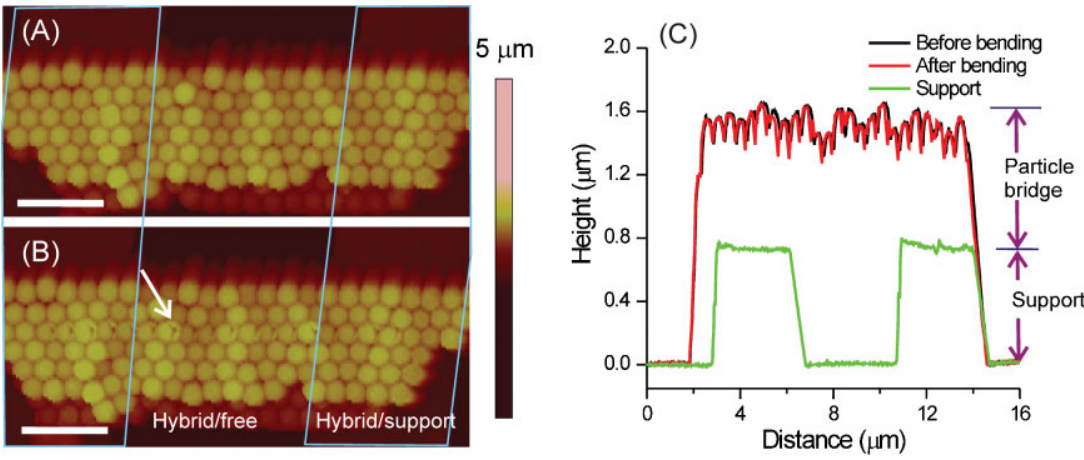

Figure 4. A,B) AFM height images and C) cross-sectional height profiles of the hybrid particle bridge before $(A, C)$ and after $(B, C)$ a bending test at $5 \mu \mathrm{N}$. Scale bars: $2 \mu \mathrm{m}$. across the width. The position of the particles along the width is indicated as $w 1-w 7$, as shown in Figure 2A. The deflection of the particles at the center of each row across the width was probed by the AFM tip. The moduli of the particle bridge (infiltrated by $30 \mathrm{LbL}$ cycles) with respect to the particle position across the width of the hybrid bridge were calculated according to Equation (2) (Figure 5A). The moduli estimated for $w 1-w 7$ fall within the same order of magnitude (0.6-1.6 GPa), which suggests that the particle bridges are insensitive to probing in the lateral direction. The particle bridges are fixed at both ends, hence higher stiffness and higher resistance of the particle bridge toward torsion are predicted, regardless of the position across the width of the bridge. ${ }^{[36]}$ The results once again show that all the individual PS-CD particles were effectively bound together by the supramolecular glues into a single hybrid microstructure with a macroscopic mechanical robustness.

Figure 5B shows the Young's moduli $E$ as determined from the particle bridges assembled with different numbers of LbL cycles. The average bending moduli $E$ were $(0.8 \pm 0.1)$, $(0.9 \pm 0.1)$, and $(1.1 \pm 0.1) \mathrm{GPa}$ for particle bridges infiltrated by 10,20 , and 30 bilayers of supramolecular glues, respectively. This result indicates that the stiffness and internal cohesion increase with the number of bilayers. To verify the dependency of the mechanical properties of the particle bridges, 3- $\mu \mathrm{m}$-wide hybrid particle bridges (with $30 \mathrm{LbL}$ cycles) were formed on a CD SAM with a 5- $\mu \mathrm{m}$ span length $L$ (Figure S5, Supporting Information). Despite an increase in the span length of the underlying supports, the particle bridges exhibited a similar modulus $E,(1.1 \pm 0.4) \mathrm{GPa}$, as the $4-\mu \mathrm{m}$ bridges $((1.2 \pm 0.4) \mathrm{GPa}$; Figure $5 \mathrm{~B})$. The results indicate that the cohesion of the hybrid particle crystal bridges is independent of the geometry of the hybrid particle crystal and its underlying support.

The relationship of span length $(L)$ and bridge deformation $(F / \delta)$ can be derived from Equation (2), as given in Equation (3):

$\frac{F}{\delta} \propto \frac{1}{L^{3}}$

Equation (3) predicts that the plots of $F / \delta$ are a factor of 1.95 smaller for the $5-\mu \mathrm{m}$ bridges than the $4-\mu \mathrm{m}$ ones. The force-deflection curves of the particle bridges with span lengths of 4 and $5 \mu \mathrm{m}$ are given in Figure 5C. A linear increase in deflection was observed with increasing applied loading for both bridges, in accordance with the behavior of classical single-beam bridges [Eq. (2)]. The maximum deflection of the particle bridge ranged from 50 to $170 \mathrm{~nm}$, which is less than $5 \%$ of the bridge span length, depending on the applied force. The experimental $F / \delta$ slopes in Figure 5C were 107 and 51 for $L=4$ and $5 \mu \mathrm{m}$, respectively, thus giving rise to a slope ratio of $\approx 2.10 \pm 0.05$. The excellent agreement with the predicted 
value is therefore consistent with our earlier assumption of a classical single beam, that is, constant $E$ and $I$ along the beam length. This allows us to predict the force-deflection behavior of particle bridges of different span lengths $L$. The theoretical $F / \delta$ behavior of particle bridges with $L=3$ and $6 \mu \mathrm{m}$ is presented in Figure $5 \mathrm{C}$ as dashed lines.

Similar measurements were repeated by using worn blunt tips as an additional experiment (tip radius $d \approx 150 \mathrm{~nm}$ ). The modulus of a bridge measured with a blunt tip was $1.7 \mathrm{GPa}$, which is in the same range as shown above for a normal sharp tappingmode tip. However, the AFM image quality produced by a blunt tip showed less detail and hence poorer resolution (Figure S3, Supporting Information).

Considering that the modulus of a dendrimer is in the range of $100-400 \mathrm{MPa},{ }^{[37]}$ it is no surprise that no stable particle bridges were formed from the particle crystals infiltrated only with Ad dendrimer (Figure S4, Supporting Information). ${ }^{[22]}$ This result is attributed to insufficient cohesion between the particles. The dramatic improvement in the mechanical robustness of the freestanding particle bridges (Figure 2) can thus be faithfully attributed to reinforcement by the LbL assembly of the hybrid organic-metallic supramolecular glues. The mechanical stability of the particle crystals was found to be further reinforced with increasing numbers of LbL cycles. It is plausible that the increase in the filling of the voids of the particle crystal by the glues improved the crosslinking of the entire particle crystal. It is noted that there is a one order of magnitude increase in the moduli of the particle bridges as compared to the dendrimer. The increment could originate from the reinforcement effects of multivalent networks of Ad dendrimers and Au-CD nanoparticles within the particle crystal and/or the high stiffness initiated by the Au-CD nanoparticles. The moduli of the particle composites, in particular after 30 cycles of supramolecular LbL assembly, are of the same order of magnitude as that of bulk PS $(E \approx 2.5 \mathrm{GPa})$, as measured by AFM-based nanoindentation (Figure 4A; for details, see Experimental Section). This finding indicates that the supramolecular composites are comparably stiff as PS. Furthermore, the results show that the micromechanical properties of the particle bridges can be tuned at will by controlling the number of bilayers of supramolecular glues applied to the particle crystal.

In some experiments, it was observed that some particle bridges failed during the bending test. Hence, the failure morphology and force curves of these failed bridges were studied in more detail. Figure 6A-C shows tappingmode AFM images and height profiles of a particle bridge $(L=5 \mu \mathrm{m})$ before and after failure. The failure of the particle bridge was recorded in situ by force spectroscopy and is represented in the force-deflection curves in Figure 6D. From particle 1 to 4, a typical reduction in the force-deflection slope was obtained when the applied force moved toward the center of the freestanding bridge. At particle 5, when the deflection exceeded $400 \mathrm{~nm}$ at $F \approx 5 \mu \mathrm{N}$, a dramatic drop in the applied force was noticed, followed by a large deflection of $>800 \mathrm{~nm}$ at decreasing force. The deformation of the failed particle 5 before and after collapse, applied repeatedly at the same position with loads ranging from 2.0 to $5.5 \mu \mathrm{N}$, is shown in Figure 6E. Linear and reproducible force-deflection curves were observed upon failure. This result shows that the particle bridge can be bent multiple times within its elastic region and prior to its ultimate strength, with no significant influence on its structural and mechanical properties. The linear part of the force-penetration curve suggests that the particle bridge is an elastic material. When the bridge was further deflected, an ultimate force occurred at $\approx 5.5 \mu N$. Such a limiting force indicates the maximum amount of force that a particle bridge can withstand. When the applied force exceeded the ultimate force, the deformation of the particle bridge could no longer recover. Hence, the structure underwent plastic deformation and did not return to its initial shape. The AFM image and height profile in Figure $6 \mathrm{~B}$ and $\mathrm{C}$ indicate that the particle bridge failed and broke into two beams at the midpoint, while the particles on the broken pieces remained connected to each other.

\section{Conclusions}

Freestanding hybrid particle bridges on 3D substrates were demonstrated by combining self-assembly of particles, supra- 
molecular LbL assembly of host- and guest-functionalized glues based on dendrimers within the particle crystal, and the transfer printing technique. An AFM-based microbending test was performed to study the mechanical properties of these unique freely suspended structures. The particle bridges showed location- and force-dependent deflection behavior, which suggests that the bridge can be described by classical beam theory. These particle bridges were found to possess a bending modulus of $0.8-1.1 \mathrm{GPa}$, which approaches the modulus of pure PS and is one order of magnitude higher than that of the dendrimers. Failure analysis on the structure indicated linear elastic behavior and a plastic deformation upon failure.

The supramolecular LbL assembly technique is not limited to freestanding line-featured bridges; it can be generally applied to the formation of freestanding structures of desired shapes and sizes. The tunable supramolecular LbL assembly method could be used to fine-tune the stiffness of the particle bridge (from semiflexible to rigid) at will. The ability to control the mechanical properties of freestanding particle bridges may initiate new perspectives for the development of the assembly and patterning of particle crystals for applications in electronic devices and M/NEMS.

\section{Experimental Section}

Materials: $\beta-C D$ heptamine ${ }^{[38]}$ and adamantyl-terminated poly(propylene imine) dendrimer of generation 5 (Ad dendrimer) ${ }^{[23]}$ were synthesized as described before. $\mathrm{N}$-[3-(Trimethoxysilyl)propyl]ethylenediamine and 1,4-phenylene diisothiocyanate were obtained from Sigma-Aldrich, Germany. PDMS Sylgard 184 and curing agent were obtained from Dow Corning, USA. Carboxylatefunctionalized PS particles $500 \mathrm{~nm}$ in size were purchased from Polysciences Inc., Germany. CD-functionalized PS particles (PS-CD), starting from the carboxylate-functionalized particles, and gold nanoparticles (Au-CD, $d \approx 3 \mathrm{~nm}$ ) were prepared as described before. ${ }^{[18,19]}$ Milli- $Q$ water with a resistivity higher than $18 \mathrm{M} \Omega \mathrm{cm}$ was used in all experiments.

Substrate and monolayer preparation: Flat and topographically patterned silicon substrates were cleaned by immersion in piranha solution (concentrated $\mathrm{H}_{2} \mathrm{SO}_{4} / 33 \% \mathrm{H}_{2} \mathrm{O}_{2}, 3: 1, \mathrm{v} / \mathrm{v}$. Warning! Piranha should be handled with caution; it is a highly corrosive oxidizing agent) for $15 \mathrm{~min}$ to form a $\mathrm{SiO}_{2}$ layer on the surface. The substrates were then sonicated in Milli-Q water and ethanol for $1 \mathrm{~min}$, and dried with $\mathrm{N}_{2}$. CD SAMs were obtained according to a published procedure. ${ }^{[15]}$ In brief, the substrates were functionalized with $\mathrm{N}$-[3-(trimethoxysilyl)propyl]ethylenediamine by gas-phase evaporation. Transformation of the aminoterminated SAMs to isothiocyanate-bearing layers was accomplished by exposure to an ethanol solution of 1,4-phenylene diisothiocyanate at $50^{\circ} \mathrm{C}$ for $2 \mathrm{~h}$. CD SAMs were finally obtained by reaction of the isothiocyanate-terminated monolayer with $C D$ heptamine in water of $\mathrm{pH} 7.5$ at $50^{\circ} \mathrm{C}$ for $2 \mathrm{~h}$.

PDMS stamps were prepared by casting a 10:1 (v/v) mixture of elastomer and curing agent against a photolithographically patterned silicon master with 3 or $4 \mu \mathrm{m}$ lines at $8 \mu \mathrm{m}$ period with a height of $750 \mathrm{~nm}$. After curing of the stamps overnight, they were mildly oxidized in an $\mathrm{O}_{2}$ plasma etcher (Tepla 300E) for $1 \mathrm{~min}$ to render them hydrophilic.

Assembly of PS-CD particles: On an oxidized PDMS stamp with $3 \mu \mathrm{m}$ lines at $5 \mu \mathrm{m}$ trenches with a height of $750 \mathrm{~nm}, 500-\mathrm{nm}$ PS-CD particles were convectively assembled into the grooves of the PDMS stamp by using a capillary-assisted deposition setup. ${ }^{[16]}$ The preformed particle array was then gently dipped in a $1 \mathrm{~mm}$ aqueous solution of Ad dendrimer for $30 \mathrm{~min}$, rinsed with water, and blown dry with $\mathrm{N}_{2}$.

Formation of particle composites: The particle composites were prepared by $\mathrm{LbL}$ assembly of Au-CD nanoparticles $(d \approx 3 \mathrm{~nm})$ and Ad dendrimers on the preassembled PS-CD particle layers, according to a published procedure. ${ }^{[22]} A$ total of 10,20 , and 30 cycles were performed in separate experiments. After each adsorption step, the substrate was blown dry with $\mathrm{N}_{2}$.

Transfer printing of particle arrays: Oxidized PDMS stamps, onto which PS-CD particles were deposited and infiltrated with Ad dendrimers or the $L b L$ assemblies, were brought into conformal contact with a flat or micropatterned CD SAM. The printing was performed in a humid environment. After removal of the stamp, the substrates were thoroughly rinsed with water and blown dry with $\mathrm{N}_{2}$.

SEM: All SEM images were taken with an HR-LEO 1550 FEF microscope.

AFM: AFM measurements were carried out using a Dimension D3100 microscope equipped with a NanoScope IVa controller and a hybrid H-153 scanner with XYZ closed-loop feedback (Veeco/ Digital Instruments (DI), Santa Barbara, CA) under ambient conditions. Silicon cantilevers (PointProbe Plus noncontact highresonance frequency (PPP-NCH) from Nanosensors, Wetzlar, Germany) were used for intermittent-contact (tapping) mode operation to obtain high-resolution images of the samples. Scan rates were varied from 0.35 to $0.5 \mathrm{~Hz}$ and free amplitude $\left(A_{0}\right)$ setpoint values were about $1.6 \mathrm{~V}$. Images of freely suspended nanostructures were taken at $85-90 \%$ of the free amplitude. Prior to the bending modulus measurements, the freely suspended nanostructures were imaged and the "point-and-shoot" function was used to pre-position the tip on a particle. A series of force-separation curves (14-20 curves per sample) with preset force trigger values were taken across the sample. The nanostructure was scanned subsequently by tapping-mode AFM. During the indentation, the X-Rotate function in the Nanoscope software version $613 \mathrm{~b}$ was set to $11^{\circ}$ in order to offset the tilt angle of the cantilever and to prevent the cantilever from plowing the surface laterally. In brief, X-Rotate provides an additional motion in the $x$-direction during indentation (for more information, see the user's guide of Nanoscope software 6.13). The effect of X-Rotate during the indentation of 500-nm PS thin film is shown in the Supporting Information (Figure S6). It shows that the most effective X-Rotate values were between 10 and $20^{\circ}$, which gave smaller indentation marks and resulted in less material accumulation on the side after indentation. For consistency, all experiments were carried out at a fixed X-rotate angle of $11^{\circ}$. The sensitivity of each cantilever, which ranged between 61 and $82 \mathrm{~nm} \mathrm{~V}^{-1}$ (average $\approx 72 \mathrm{~nm} \mathrm{~V}^{-1}$ ), was calibrated on a hard substrate (i.e., silicon) after the experiment. The force applied on the sample was determined by the deflection of the cantilever multiplied by the spring constant 
value of the cantilever. The spring constants of the cantilevers were calibrated according to Sader's method. ${ }^{[39]}$ The spring constant values obtained were in the range of $25-31 \mathrm{~N} \mathrm{~m}^{-1}$ with a mean value of $28 \mathrm{~N} \mathrm{~m}^{-1}$. Data conversion was carried out with Nanoscope software version $6.13 \mathrm{~b}$ after inserting the corrected sensitivity obtained on the silicon surface and using the calibrated spring constant value. For the indentation of the PS thin film, the modulus was calculated according to Oliver and Pharr's method. ${ }^{[40]}$ For the morphology of the failed nanostructure, the raw data image was used throughout the analysis and the contrast of the images was adjusted accordingly.

\section{Acknowledgements}

The authors gratefully acknowledge the financial support by the Council for Chemical Sciences of the Netherlands Organization for Scientific Research (NWO-CW; Vidi Vernieuwingsimpuls grant 700.52.423 to J.H. and NWO middelgroot grant to D.N.R. and G.J.V.), and by the Dutch Polymer Institute (grant \#DPI-510 grant to G.J.V.).

[1] E. Menard, M. A. Meitl, Y. G. Sun, J. U. Park, D. J. L. Shir, Y. S. Nam, S. Jeon, J. A. Rogers, Chem. Rev. 2007, 107, 1117.

[2] H. G. Craighead, Science 2000, 290, 1532.

[3] T. Namazu, Y. Isono, T. Tanaka, J. Microelectromech. Syst. 2000, 9 , 450.

[4] A. San Paulo, N. Arellano, J. A. Plaza, R. R. He, C. Carraro, R. Maboudian, R. T. Howe, J. Bokor, P. D. Yang, Nano Lett. 2007, 7, 1100.

[5] F. Hua, T. Cui, Y. M. Lvov, Nano Lett. 2004, 4, 823.

[6] H. Xu, R. Hong, T. Lu, O. Uzun, V. M. Rotello, J. Am. Chem. Soc. 2006, 128, 3162.

[7] W. T. S. Huck, A. D. Stroock, G. M. Whitesides, Angew. Chem. 2000, 112, 1100; Angew. Chem. Int. Ed. 2000, 39, 1058.

[8] C. Lu, I. Donch, M. Nolte, A. Fery, Chem. Mater. 2006, 18, 6204.

[9] N. Malikova, I. Pastoriza-Santos, M. Schierhorn, N. A. Kotov, L. M. Liz-Marzan, Langmuir 2002, 18, 3694.

[10] C. Y. Jiang, S. Markutsya, Y. Pikus, V. V. Tsukruk, Nat. Mater. 2004, 3, 721.

[11] A. A. Mamedov, N. A. Kotov, M. Prato, D. M. Guldi, J. P. Wicksted, A. Hirsch, Nat. Mater. 2002, 1, 190.

[12] A. A. Mamedov, N. A. Kotov, Langmuir 2000, 16, 5530.

[13] P. Podsiadlo, A. K. Kaushik, E. M. Arruda, A. M. Waas, B. S. Shim, J. D. Xu, H. Nandivada, B. G. Pumplin, J. Lahann, A. Ramamoorthy, N. A. Kotov, Science 2007, 318, 80.
[14] R. H. A. Ras, T. Ruotsalainen, K. Laurikainen, M. B. Linder, O. Ikkala, Chem. Commun. 2007, 1366.

[15] P. Maury, M. Péter, O. Crespo-Biel, X. Y. Ling, D. N. Reinhoudt, J. Huskens, Nanotechnology 2007, 18, 044007.

[16] X. Y. Ling, I. Y. Phang, D. N. Reinhoudt, G. J. Vancso, J. Huskens, Int. J. Mol. Sci. 2008, 9, 486.

[17] X. Y. Ling, D. N. Reinhoudt, J. Huskens, Langmuir 2006, 22, 8777.

[18] O. Crespo-Biel, B. Dordi, D. N. Reinhoudt, J. Huskens, J. Am. Chem. Soc. 2005, 127, 7594.

[19] X. Y. Ling, L. Malaquin, D. N. Reinhoudt, H. Wolf, J. Huskens, Langmuir 2007, 23, 9990.

[20] O. Crespo-Biel, B. Dordi, P. Maury, M. Péter, D. N. Reinhoudt, J. Huskens, Chem. Mater. 2006, 18, 2545.

[21] X. Y. Ling, D. N. Reinhoudt, J. Huskens, Chem. Mater. 2008, 20, 3574.

[22] X. Y. Ling, I. Y. Phang, W. Maijenburg, H. Schönherr, D. N. Reinhoudt, G. J. Vancso, J. Huskens, Angew. Chem. 2009, 121, 1001; Angew. Chem. Int. Ed. 2009, 48, 983.

[23] J. J. Michels, M. Baars, E. W. Meijer, J. Huskens, D. N. Reinhoudt, J. Chem. Soc. Perkin Trans. 2 2000, 1914.

[24] V. Sazonova, Y. Yaish, H. Ustunel, D. Roundy, T. A. Arias, P. L. McEuen, Nature 2004, 431, 284.

[25] T. W. Tombler, C. W. Zhou, L. Alexseyev, J. Kong, H. J. Dai, L. Lei, C. S. Jayanthi, M. J. Tang, S. Y. Wu, Nature 2000, 405, 769.

[26] J. P. Salvetat, G. A. D. Briggs, J. M. Bonard, R. R. Bacsa, A. J. Kulik, T. Stockli, N. A. Burnham, L. Forro, Phys. Rev. Lett. 1999, 82, 944.

[27] B. Wu, A. Heidelberg, J. J. Boland, Nat. Mater. 2005, 4, 525.

[28] A. Kis, S. Kasas, B. Babic, A. J. Kulik, W. Benoit, G. A. D. Briggs, C. Schönenberger, S. Catsicas, L. Forro, Phys. Rev. Lett. 2002, 89.

[29] H. Ni, X. D. Li, Nanotechnology 2006, 17, 3591.

[30] L. Yang, K. O. Van der Werf, C. F. C. Fitie, M. L. Bennink, P. J. Dijkstra, J. Feijen, Biophys. J. 2008, 94, 2204.

[31] S. Y. Gu, Q. L. Wu, J. Ren, G. J. Vancso, Macromol. Rapid Commun. 2005, 26, 716 .

[32] Q. Xiong, N. Duarte, S. Tadigadapa, P. C. Eklund, Nano Lett. 2006, 6, 1904.

[33] L. J. Niu, X. Y. Chen, S. Allen, S. J. B. Tendler, Langmuir 2007, 23, 7443.

[34] G. Decher, Science 1997, 277, 1232.

[35] M. J. W. Ludden, D. N. Reinhoudt, J. Huskens, Chem. Soc. Rev. 2006, 35, 1122.

[36] For a rectangular beam of length $L$ and Poisson ratio $v$, the torsional spring constant $\left(k_{t}\right)$ divided by the normal spring constant $\left(k_{\mathrm{n}}\right)$ is equal to $2 L^{2} / 3(1+v)$. Hence, $k_{\mathrm{t}} \gg k_{\mathrm{n}}$.

[37] H. Shulha, X. Zhai, V. V. Tsukruk, Macromolecules 2003, 36, 2825.

[38] M. W. J. Beulen, J. Bügler, B. Lammerink, F. A. J. Geurts, E. Biemond, K. G. C. van Leerdam, F. C. J. M. van Veggel, J. F. J. Engbersen, D. N. Reinhoudt, Langmuir 1998, 14, 6424.

[39] J. E. Sader, I. Larson, P. Mulvaney, L. R. White, Rev. Sci. Instrum. $1995,66,3789$.

[40] W. C. Oliver, G. M. Pharr, J. Mater. Res. 1992, 7, 1564.

Received: January 13, 2009

Revised: February 24, 2009

Published online: April 16, 2009 